CENTRO NACIONAL DE ACELERADORES (CNA) SEVILLA, SPAIN

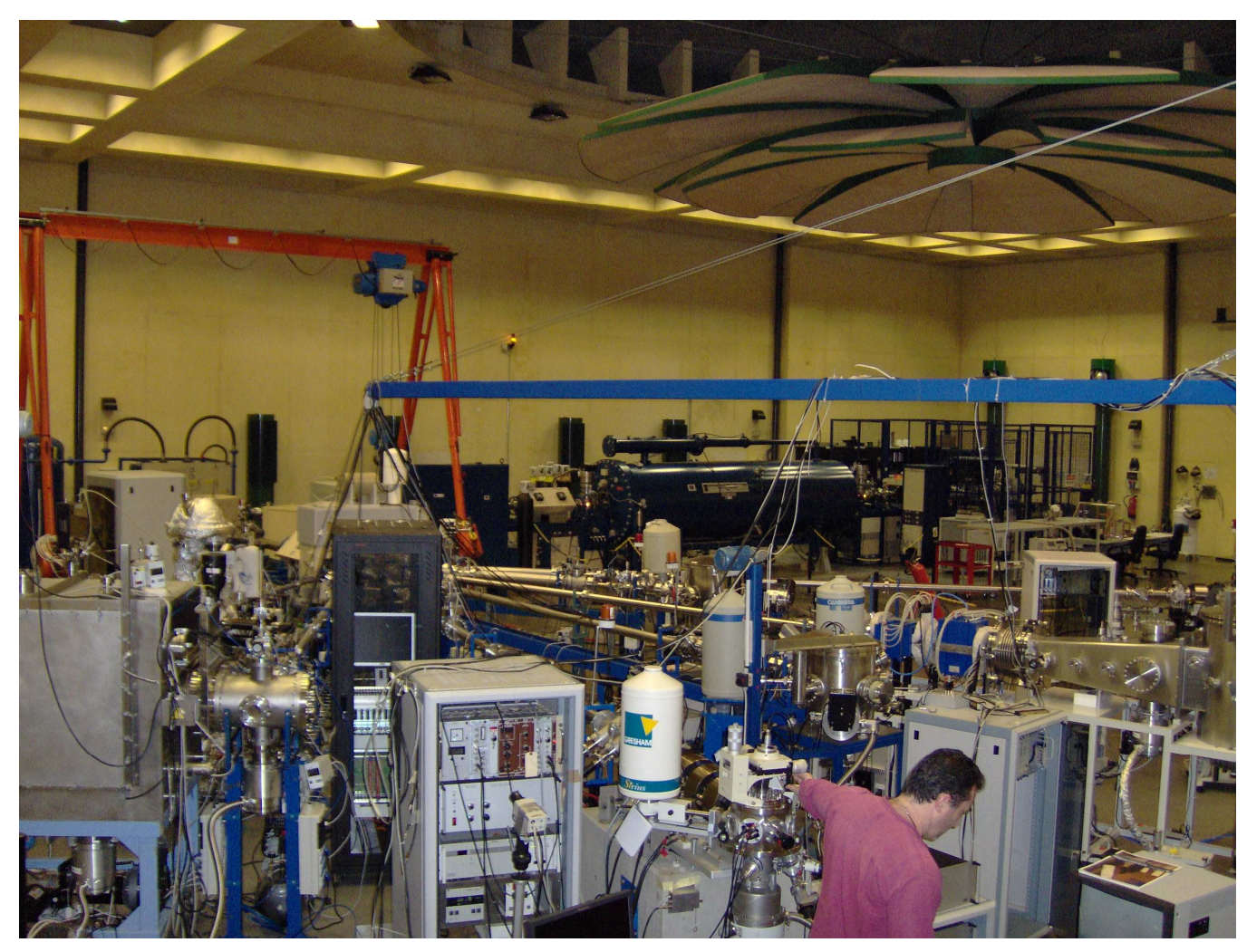

\title{
Introduction
}

The National Accelerator Centre (CNA) is a Spanish joint institution which, since its creation in 1998, has the mission of carrying out research in particle accelerators and its multiple applications. This article aims to perform a summary of the structure and the facilities of the Centre, while it also contains information about the most recent lines of research with an accent on those belonging to the nuclear physics field. In this way, we want to show the capabilities of CNA, in order to promote collaborations with the scientific and technological communities. The CNA is recognized as an Spanish Singular Scientific and Technological Facility, and it is a users-oriented laboratory, open to the Spanish and the international scientific community belonging to universities, public research institutions, public and private companies, hospitals or other institutions that require the use of the facilities.

The CNA is placed in Seville, in the "Isla de la Cartuja" Tecnological Park, and was originated from an agreement among the University of Seville, the Regional Government of Andalucía (Junta de Andalucía) and the Spanish High Council of Scientific Research (CSIC). The Governing Board of the Centre, constituted by representatives of the three institutions, indicates the general strategy and 
policy issues of the Centre, which are carried out by the CNA Director. There is also a Scientific Committee, with scientists proposed by the three institutions, that advice the Director on the scientific strategy and regulate the use of CNA infrastructure. The scientific personnel of the Centre are distributed in Research Units, which are organized around the different infrastructures and capabilities of the Centre. Besides that, there is an Administration and Technical Unit that provides administrative and technical support to the Centre and to the external CNA users. The present staff of the centre is about 40 persons, including researchers, technicians, administration and postgraduates.

\section{Main Infrastructures of CNA}

CNA is a pioneering centre in Spain in the field of applications of particle accelerators for research. It has three particle accelerators: a 3 MV van de Graaff tandem accelerator, a cyclotron that supplies $18 \mathrm{MeV}$ protons and $9 \mathrm{MeV}$ deuterons, and a $1 \mathrm{MV}$ Cockcroft-Walton tandem accelerator (called Tandetron) which is indeed a mass spectrometer. These accelerators provide a series of unique research tools to the scientific community: Ion Beam Analysis (IBA) for material characterization, modification and irradiation of materials, production of radionuclides for Positron Emission

Tomography (PET) and Accelerator Mass Spectrometry (AMS) that includes a ${ }^{14} \mathrm{C}$ dating service using AMS. At CNA, there are also research laboratories for sample preparation that facilitate the application of the presented techniques.

\section{MV Tandem Accelerator.}

The first accelerator at CNA is a Pelletron $3 \mathrm{MV}$ Tandem, made by National Electrostatics Corporation (NEC), and installed in 1998. It is primarily focused on material characterization and modification by means of IBA techniques (PIXE, RBS, ERDA, NRA and PIGE). 


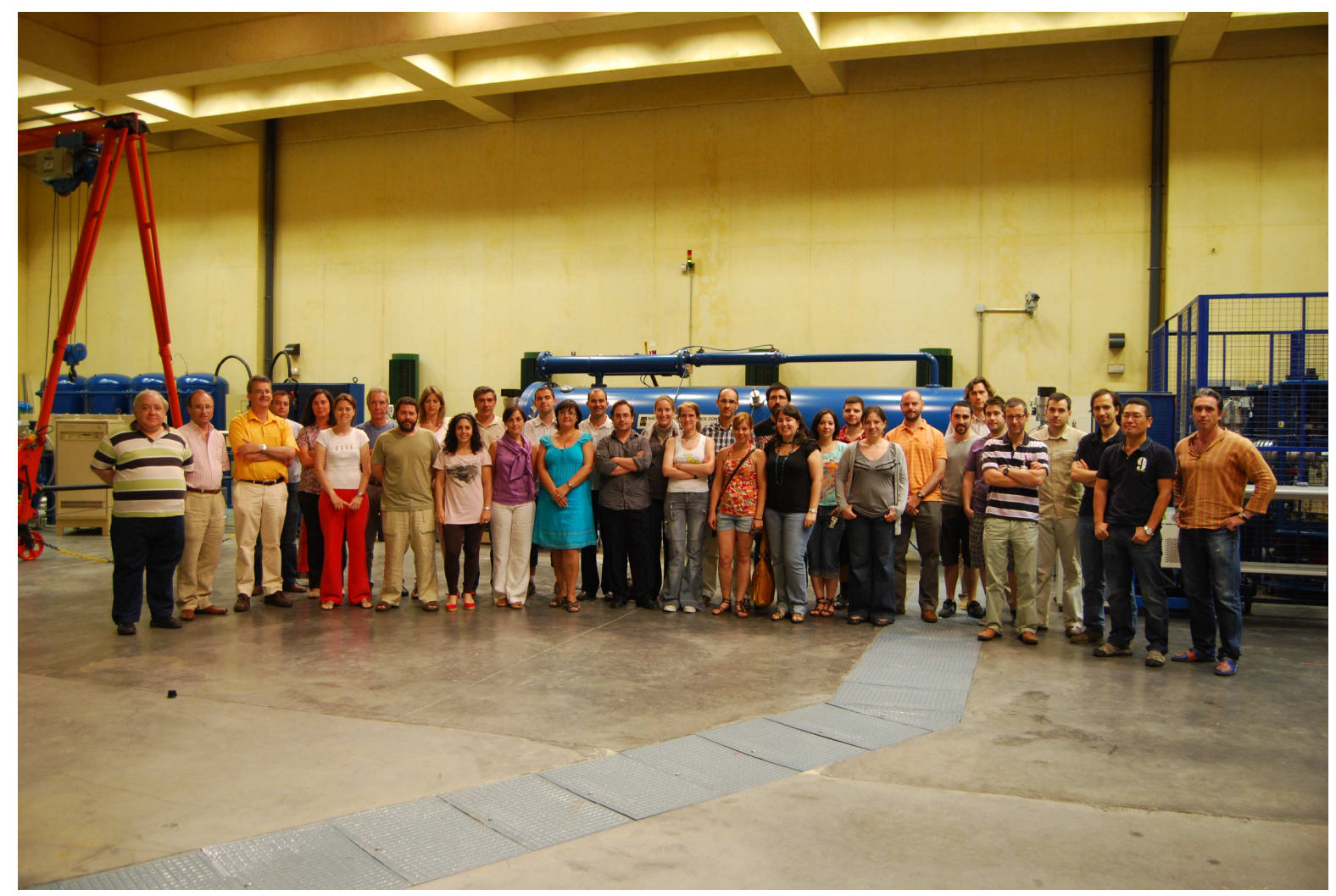

Fig. 1: Some of the CNA staff, in front of the 3 MV Tandem accelerator at CNA.

In this device, ions can be produced by three ion sources. The first one is based on radiofrequency techniques (Alphatross) and generates negative ions from gases $(\mathrm{H}, \mathrm{He}, \mathrm{N} \ldots)$. There is also a caesium sputtering source (SNICS) that produces negative ions from solid samples. The most recent source is a Duoplasmatron source, which is very reliable and provides a high-brightness proton beam. The sources are connected by ports to an injection magnet that selects the desired ion mass. Typical beam intensities produced are up to $20 \mu \mathrm{A}$ for protons.

In the two-pellet chain tandem accelerator, the selected ions are accelerated with a maximum terminal voltage of $3 \mathrm{MV}$. The beam line that is situated after the accelerator includes several instruments for focusing, steering and monitoring the beam. At the line end, the beam can pass directly to the $0^{\circ}$ line or it can be deflected by the switcher magnet towards one of the beam lines. At the present time, there are six available beam lines to characterize and to modify materials, as well as for Nuclear Physics research.

$-30^{\circ}$ Beam Line: Nuclear Physics Beam Line.

It is equipped at its end with a $1.0 \mathrm{~m} \times 1.0 \mathrm{~m} \times 1.5 \mathrm{~m}$ vacuum chamber with a large lateral port, capable of housing detectors of large dimensions. Inside the chamber, a special table is installed which can be moved up and down and has a set of drillings, allowing versatile mounting of detectors and related instrumentation. The main objective is to perform different tests of detectors and related instrumentation, including electronics. 
-15Beam Line: Microbeam Chamber.

With the microprobe focusing system it is possible to form a spot of a few microns on the specimen. Elemental maps that provide the spatial distribution concentration in the samples are obtained with the scanning mode. The scanning coils allow a maximum scanning area of $2.5 \mathrm{~mm} \times 2.5 \mathrm{~mm}$ for 3 $\mathrm{MeV}$ protons.

\section{Beam Line: Multipurpose IBA Chamber.}

This target chamber has been designed to carry out RBS, PIXE, NRA and PIGE experiments simultaneously. A set of gamma, particle and X-Ray detectors is used to combine all these IBA techniques.

\section{$+15^{\circ}$ Beam Line: Irradiation Chamber.}

This home-made scattering chamber has been designed to allow the irradiation of large areas (16 $\mathrm{cm} \times 20 \mathrm{~cm}$ ) by raster scanning of the beam through magnetic deflection. It is a movable beam line, in such a way that the system can be easily transported to the cyclotron when irradiation with high energy protons is required. It allows also ionic implantation at energies higher than with conventional small electrostatic accelerators.

\section{$+30^{\circ}$ Beam Line: Channelling Chamber.}

This line is primarily devoted to channelling analysis of crystalline samples. A well-defined parallel beam is obtained with a telescopic system formed by two slit assemblies. The beam line also includes a BPM and a Faraday cup. The chamber is equipped with two particle detectors and an XRay detector.

\section{$+45^{\circ}$ Beam Line: External Beam.}

This line is mainly used in Art and Archaeometry studies. Due to the frequent inhomogeneous nature of this kind of objects, the use of external ion beam analysis (with a good resolution) exhibits numerous advantages. With the idea of achieving good spatial resolutions ( $60 \mu \mathrm{m})$, a series of elements have been installed, including a precision four-jaw object slit and an exit nozzle set with micrometer adjustment.

Associated to the Tandem, there is a service of IBA measurements that provides analyses of samples sent by the users, by means of PIXE, RBS, NRA and other IBA techniques. Although the samples which are measured by IBA techniques do not need generally exhaustive preparation, the 3 MV Tandem accelerator has an associated laboratory with all the necessary equipment to carry out different treatments to the samples before they are studied.

\section{Cyclotron}


The cyclotron was the second particle accelerator installed at CNA (year 2004). The CNA cyclotron, the Cyclone 18/9, was manufactured by the company IBA (Belgium) and accelerates protons and deuterons to 18 and $9 \mathrm{MeV}$ respectively. The extracted maximum beam intensities in the internal target ports are $80 \mu \mathrm{A}$ for protons and $35 \mu \mathrm{A}$ for deuterons. Besides that, the Cyclone 18/9 allows the simultaneous bombardment with the same particle of one or two targets that are located in opposite positions (Dual Beam Mode).

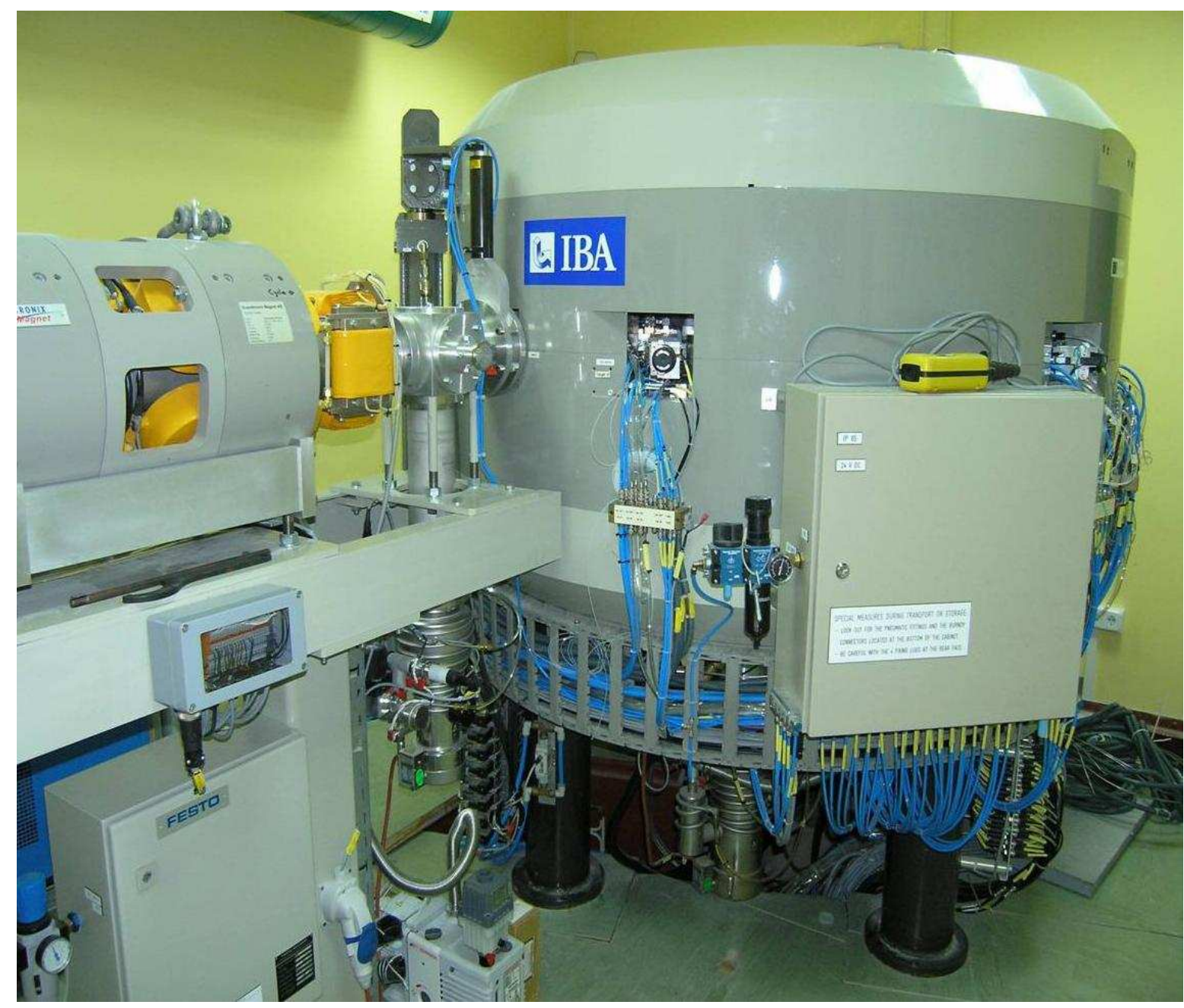

Fig. 2: Cyclotron at CNA. The external beam line is seen to the left.

Seven out of the eight targets are devoted to the production of positron emitters. Thus, CNA offers the possibility to produce the most frequent radioisotopes employed in the imaging modality Positron Emission Tomography (PET). In the last target port there is an exit line (Experimental Beam Line) that transports the beam line to a second vault. A reaction chamber devoted to the irradiation of technological materials is located in this second room with two major purposes: to study the effects of $18 \mathrm{MeV}$ proton irradiation on the behavior of electronic devices for space applications and to complement the analysis of materials using the $3 \mathrm{MV}$ tandem accelerator with the PIXE measurements at high energy (HIPIXE). The cyclotron provides a unique energy for protons and deuterons, but a series of degraders may be introduced in order to decrease the energy value. The 
singularity of this existing equipment at CNA means this is the highest energy facility in Spain for material irradiation.

The Cyclotron accelerator has associated a unit with several radiopharmaceutical laboratories which were designed to comply with Good Manufacturing Practices (GMP) regulations. At the present time, the unit is composed of an "F-FDG Production Lab" and a "Research Lab" where PET radiopharmaceuticals are prepared for preclinical studies. These two laboratories are complemented by a quality control laboratory. The production of [ $\left.{ }^{18} \mathrm{~F}\right] \mathrm{FDG}$ at CNA began in 2005 as part of a contract agreement with IBA Molecular in a FDG Production Lab equipped with two hot cells that hold two double GE Tracerlab FX-FDG synthesis modules. The Research Lab has five hot cells where radiopharmaceuticals that trace metabolic or functional processes $\left(\left[{ }^{18} \mathrm{~F}\right] \mathrm{F}-\mathrm{DOPA},\left[{ }^{18} \mathrm{~F}\right] \mathrm{FLT}\right.$, $\left[{ }^{18} \mathrm{~F}\right] \mathrm{FMISO},\left[{ }^{18} \mathrm{~F}\right] \mathrm{F} 4 \mathrm{~B}$, sodium $\left[{ }^{18} \mathrm{~F}\right]$ fluoride, $\left[{ }^{11} \mathrm{C}\right]$ choline, $\left[{ }^{13} \mathrm{~N}\right]$ ammonia) are synthesized.

Associated also to the cyclotron accelerator there is a research laboratory devoted to the application of PET techniques in pre-clinical research. This laboratory is equipped with a small-animal PET scanner made by Philips (model Mosaic) based on 14,456 GSO crystals (gadolinium orthosilicate activated with cerium) arranged in 52 rings of 278 crystals each. It has also a preclinical micro CT system (made by Bioscan, model Nano CT) installed by Philips Medical Systems. This Nano CT system is a helical CT scanner obtaining images with a spatial resolution higher than $200 \mu \mathrm{m}$. This unit is used to obtain computed tomography (CT) images with X-rays from small animals and objects of technological or archaeological interest. Sequential image acquisition with both techniques (PET/CT) in experimental animals is possible and a unique multimodality PET/CT image is finally obtained, combining PET metabolic and CT morphologic information.

\section{MV Tandetron for AMS}

Since February 2006, a 1 MV multielement compact AMS facility, SARA (Spanish Accelerator for Radionuclides Analyses), is fully operative at CNA. This system was acquired to High Voltage Engineering Europe, becomes the only facility of AMS in Spain, and follows the actual tendency of using compact AMS systems with low terminal voltages because they have demonstrated to reach detection limits as low as the high-energy facilities for many of the most common radionuclides in AMS.

The SARA device is equipped with low and high-energy magnets specially designed to support the analysis of heavy elements such as ${ }^{129} \mathrm{I}$ and $\mathrm{Pu}$ isotopes. The ion source system is equipped with a carousel with capacity for 200 samples, from which an automatic sample changer selects the cathode following the sequence of a batch. The final detection of the particles is performed with a two-anode gas ionization chamber provided with a $75 \mathrm{~nm}$ thickness silicon nitride window. 


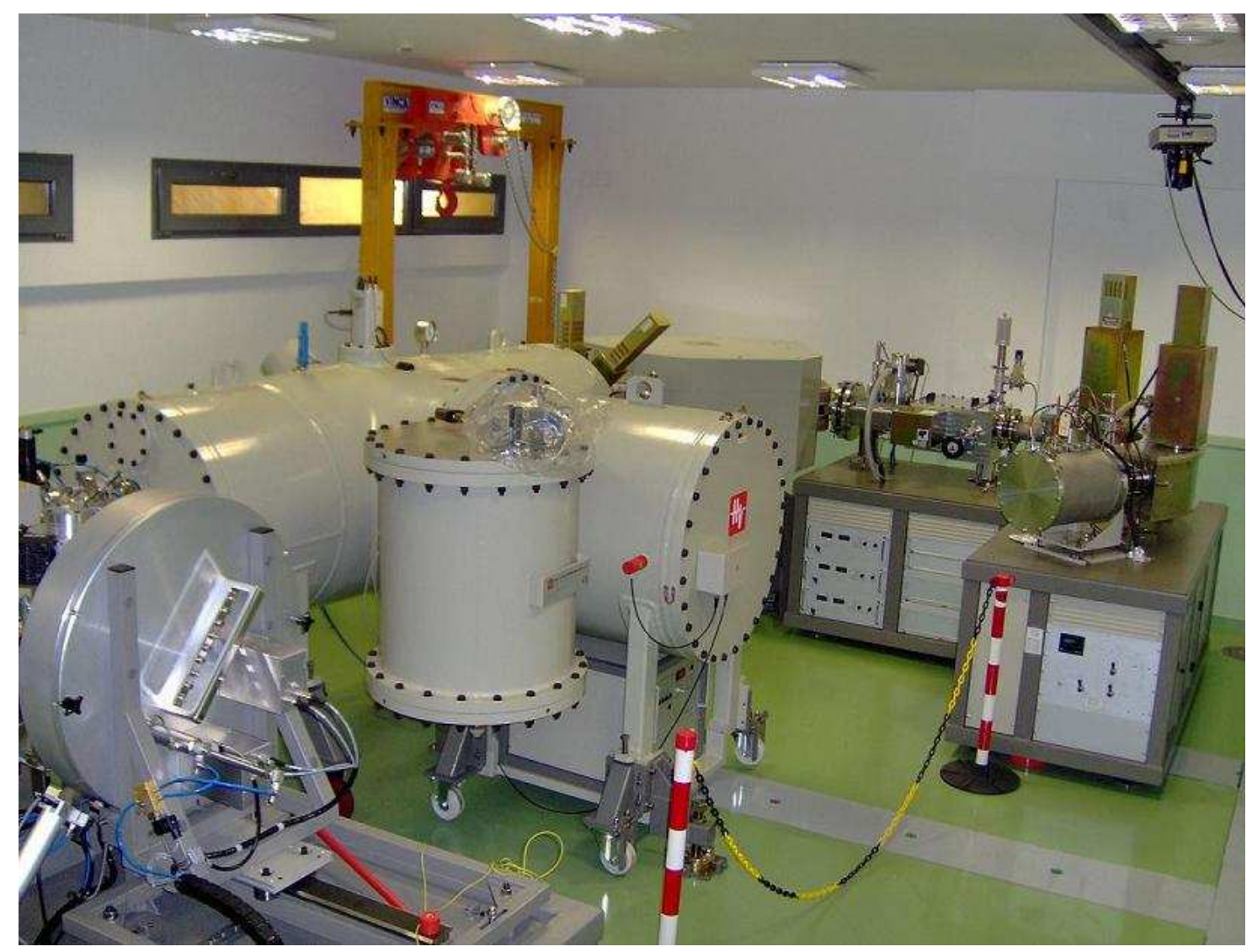

Figure 3: SARA device at CNA. The carousel with the samples is on the left, the $1 \mathrm{MeV}$ Tandetron accelerator in the centre and the gas ionization chamber is on the right.

The SARA device was originally designed for the measurement of ${ }^{10} \mathrm{Be},{ }^{14} \mathrm{C},{ }^{26} \mathrm{Al},{ }^{129} \mathrm{I}$ and isotopes of $\mathrm{Pu}$. In the last years, active lines have focused on the analysis of samples for ${ }^{14} \mathrm{C},{ }^{26} \mathrm{Al},{ }^{129} \mathrm{I}$ and $\mathrm{Pu}$ isotopes, but tests to study the team's ability to detect new radioisotopes as the ${ }^{41} \mathrm{Ca},{ }^{36} \mathrm{Cl} .{ }^{236} \mathrm{U}$ and ${ }^{237} \mathrm{~Np}$ have begun. According to the matrix in which radioisotopes are located, different sample preparation methods are used, whose main objective is to isolate the isotope of interest from other possible interferents previous to their measurement by AMS. These sample preparation methods are applied in an "AMS radiochemical laboratory" equipped with all the facilities needed for the application to the samples under analysis.

Associated to this facility, there is a Radiocarbon Dating Service $\left({ }^{14} \mathrm{C}\right)$ at CNA where samples (archaeological, artistic, historical, etc.) can be dated by measuring their ${ }^{14} \mathrm{C}$ content. This service includes a fully-equipped laboratory for sample preparation. In 2010, more than 300 samples were dated by ${ }^{14} \mathrm{C}$, providing a service to a large national and international community. The capability of this laboratory will be enhanced in the very near future with new grafitization lines which allow the automatization of the graphitization process applied to each sample. 


\section{RESEARCH LINES AT CNA}

The research performed at CNA is carried out by five operative research units: Analysis Techniques and Materials Modification with Ion Beams Research Unit (IBA Unit), Basic Nuclear Physics Research Unit (FNB Unit), Analysis of Physical Environment Pollutants using techniques based on Accelerators Research Unit (ACOMETA Unit), Accelerator Mass Spectrometry Research Unit (AMS Unit), and Cyclotron Research Unit. This section includes in a comprehensive manner the most recent research of CNA research units, which is done usually in collaboration with external researchers.

\section{Materials Science}

The use of 3-MV tandem accelerator has been especially important to investigate in CNA many problems related to materials science. A large number of studies have been aimed at studying the composition of the samples and their correlation with the physical properties using various analytical techniques available to the CNA, such as RBS, PIXE, NRA, PIGE and ERDA. In this sense, we can emphasize the characterization of thin films of hydrogenated diamond-like carbon doped with fluorine, such as coatings that are proposed to improve wear and antiseptic properties of ultrahigh molecular weight polyethylene used in hip and knee implants; the study of mixed oxide films Ti-Tb (Eu)-O, Zn-Tb-O and Si-Tb (Er)-O with optical functionality and potential application as fluorescent coatings; the analysis of $\mathrm{SiO}_{2}$ based films doped with fluorine, which are proposed as stain resistant coatings for ophthalmic lenses due to its hydrophobic character, and the characterization of amorphous $\mathrm{SiC}$ samples doped with $\mathrm{H}$, of great interest due to their optoelectronic and mechanical properties. These works are done in collaboration with CSIC materials science institutes.

\section{Environmental Sciences}

The 3 MV Tandem accelerator and the external line of the Cyclotron has found also many applications in environmental science studies. We can highlight, as an example, the analysis performed in particles with a high concentration of activity (hot particles). In particular we have established the elemental U / Pu ratio for particles from Palomares (Spain) and Thule (Greenland) by PIXE. Pollution in these two scenarios is a result of aircraft accidents in which nuclear bombs were released and they exploded upon impact with the ground, releasing part of the nuclear fuel they contained. The characterization of these particles is essential to assess what radioecological risks may occur in the affected areas. This is done in collaboration with IAEA-MEL (Monaco) and CIEMAT.

The microPIXE technique has also been used for chemical and mineralogical speciation of soils polluted by trace elements. The technique has showed to be powerful enough to prove the association between trace elements such as $\mathrm{As}, \mathrm{Pb}, \mathrm{Cu}$ y $\mathrm{Zn}$ and several mineral phases such as iron oxy-hydroxides, chalcopyrite and galena. As a matter of fact, this technique has been proposed 
as an alternative or complementary in chemical-mineralogical studies of soils polluted by trace elements. This is done in collaboration with the Universities of Seville and Huelva.

\section{Cultural Heritage}

In the field of Cultural Heritage, non-destructive analyses of different objects have been carried out, using both the vacuum chamber as the external beam line of the $3 \mathrm{MV}$ Tandem accelerator. Elementary composition of Tartessic gold jewelry has been investigated. Interesting results have been obtained also in gilded items with silver and bronze substrates. Using a combination of RBS and PIXE techniques it was possible to determine the composition and thickness of the gold layer and the gilded metallic piece, as well as to find out the gilded method used during its fabrication.

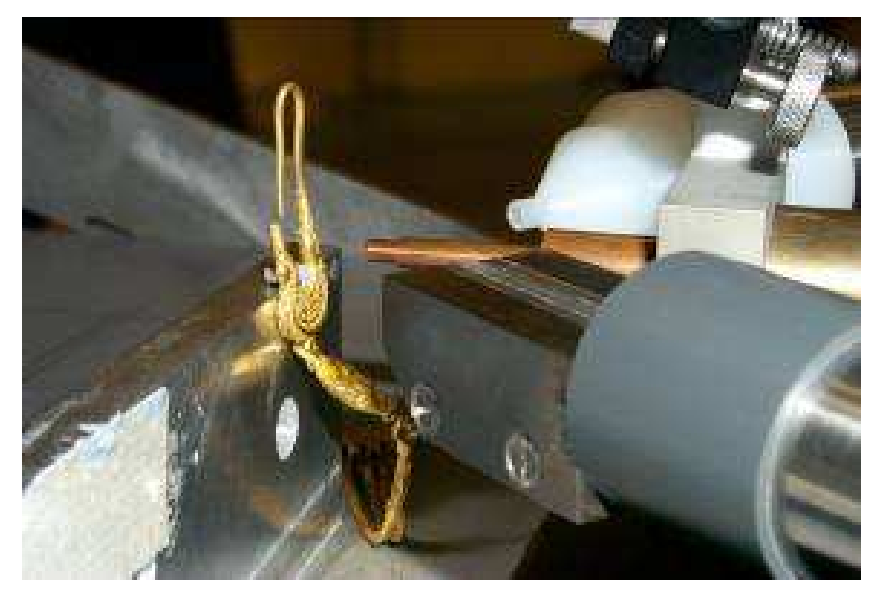

Fig 4: A piece of Tartesic jewelry (Bronze age) analyzed in the Tandem external beam line.

Another recently opened research line is the study of paleobiologic materials (bones, ivory, shells, turtles, snails, etc) in which the presence of pollutants (lead, iron, manganese, etc) as well as elements characteristics from the diet (copper, zinc, barium, etc) was determined. This is done in collaboration with the Andalusian Institute of Historical Heritage (IAPH).

\section{Irradiation}

During the last two years, irradiation studies have been carried out at CNA. This includes studies to test the reliability of devices that can be used in critical atmosphere of radiation, such as the space environment and particle physics experiments. These tests are performed with charged particles at the Tandem Accelerator or at the Cyclotron, depending on the irradiation energy required. The usual working-lines are the vacuum microprobe, the irradiation and implantation line and the cyclotron external beam line. The reliability of a device under radiation can be studied, among others, based on the possible impact of a single ionizing particle (Single Event Effects, SEE) or according to a given dose of radiation accumulated (Total Ionization Dose, TID).

In this field, we have studied the production of circuit failures "in vivo" due to different particles radiation, varying the energy and fluence. Circuits of different technologies have been specially designed and monitored, and on these circuits, total-dose and microdosimetry tests have been 
carried out to detect points of failure. Experiments in collaboration with the Engineering school of the University of Seville have been performed to generate Singular Events Upset (SEU) in a digital circuit with oxygen microbeams, ranging from 14 to $18 \mathrm{MeV}$, using the vacuum microprobe in the tandem accelerator. In collaboration with the Institute of Physics of Cantabria (IFCA), dynamic and static proton irradiation on several series of optical fiber sensors has been carried out. These sensors have been designed to be used in particle physics experiments as part of detectors for the "Large Hadron Collider" at CERN. Irradiations were performed in air, maintaining better than $10 \%$ homogeneity in the current density and calibrating the system to achieve a complete irradiation of the samples surface. Finally, in collaboration with the company ALTER, photodiodes irradiation tests have been completed as part of the evaluation required to be used in aerospace.

\section{Nuclear Physics}

The nuclear physics research unit works in collaboration with European institutions, such as CEASaclay and GANIL, that are leaders in the development of beam tracking detectors. These detectors can monitor the particle beams that will be produced in exotic beam facilities such as FAIR and SPIRAL-2. On this frame, the FNB group of the CNA is investigating on particle detectors that can track relatively intense beams of exotic nuclei at low energies. It has studied detectors based on electron emission foils coupled to gas detectors at low pressure, to improve the performance at the high counting rate that will be provided by future accelerators. Prototypes of secondary electrons detectors (SED) at low pressure are built and tested with our collaboration.

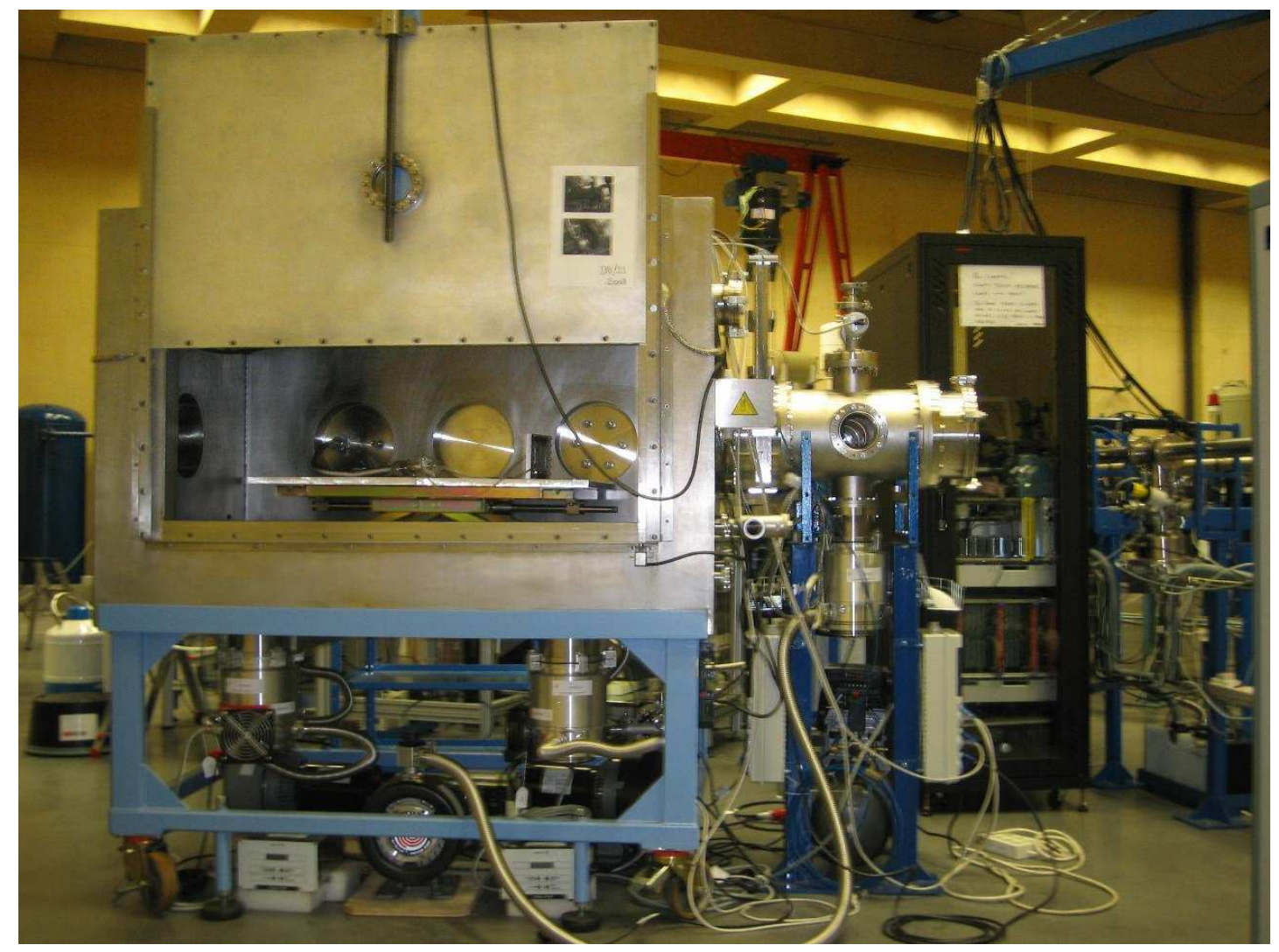

Figure 5: Beam tracking chamber at CNA, containing a model of the SED detector to study gas flow. 
Development of fast electronics systems is also necessary to process the outputs signals from particle tracking detectors. Electronics front-ends must filter, amplify and shape high count rate current signals from this kind of detectors. So, front-ends research and development defines a new field of working for the FNB Unity, in collaboration with several research groups. An international collaboration with CEA-Saclay (Saclay, France) in 2009 and 2010, has allowed implementing the first electronics front-end. It is a mixed signal preamplifier to process current/charge signals from Secondary Electron Detectors (SED).

The FNB research group of CNA is also part of the European network DITANET: "Diagnostic and Techniques for future particle Accelerators NETwork", a Marie Curie Initial Training Network (ITN) supported by the EU. This network consists of 10 major Nuclear Physics research centers in Europe, including leading universities and industries that work on this field. The aim of this project is to develop diagnostic techniques for future particle accelerator facilities and to train PhD students and young researchers within European research centers. Based on this partnership, non-interceptive optical monitors based on gas fluorescence (Fluorescence Profile Monitors, FPM) have been tested, in collaboration with CIEMAT, at the external beam line of the Cyclotron at CNA. These profiles have been designed for use on the IFIMIF project: the International Fusion Materials Irradiation Facilities, currently under development.

Within an international collaboration, led by Granada University, a measurement has been carried out at CNA on the half life of the nucleus ${ }^{8} \mathrm{Li}$. This nucleus was produced by a transfer reaction ${ }^{7} \mathrm{Li}(\mathrm{d}, \mathrm{p})^{8} \mathrm{Li}$, using a deuteron beam of $2 \mathrm{MeV}$ energy and $40 \mathrm{nA}$ intensity. A system was used to pulse the beam of the CNA tandem. The results allowed increasing the precision of the lifetime of this nucleus in a factor 2.5 .

Besides the different research lines on nuclear instrumentation, the nuclear physics group of CNA performs also research on the measurements, experimental analysis and theory of nuclear reactions with both stable and exotic nuclei. On this frame, were performed nuclear reaction experiments in the FNB line and analyzed the data collected,. Presently, we are working on the isotopes of the Helium $(\mathrm{He}){ }^{4,6,8} \mathrm{He}$ and the Lithium ( $\left.\mathrm{Li}\right){ }^{6,7,8,9,11} \mathrm{Li}$. Experiments have been carried out in international facilities, such as TRIUMF (Canada), Louvain la Neuve (Belgium), GANIL (France) and RIBRAS (Brasil). The FNB group has also participated in research in Neutron physics for Nuclear Astrophysics and Nuclear Power Generation through the participation in the n_TOF collaboration (neutron time-of-flight) at CERN and the development of a neutron line in the CNA. The measurements at $n \_T O F$ are neutron capture and neutron fission cross sections of several isotopes with application to nuclear astrophysics and nuclear power generation. In the CNA context, this line of research is also devoted to the study of the production of neutrons with maxwellian energy spectrum, as such of the stellar sites. 
The SARA system has been optimized to measure the radionuclide ${ }^{129} \mathrm{I}$, from the point of view of background levels and sensitivity, obtaining results which confirm the viability of this facility to make routine measurements of ${ }^{129} \mathrm{I}$ at environmental levels. A radiochemical method for sample preparation has been developed, that can be adapted to different environmental matrices. After that, over 300 environmental samples have been prepared following this radiochemical procedure and have been measured by AMS, including lichens, algae, peat bogs, air filters and reference materials from international agencies. These results have played essential roles in different radioecological or oceanographic studies performed in collaboration with institutions like IAEA-MEL and University of Lund.

The AMS technique has been also applied for the independent determination of ${ }^{239} \mathrm{Pu}$ and ${ }^{240} \mathrm{Pu}$ in environmental samples. These radionuclides are traditionally measured by alpha-spectrometry with two important limitations: the relatively high limit of detection and the impossibility of obtaining information on the ${ }^{240} \mathrm{Pu} /{ }^{239} \mathrm{Pu}$ atomic ratio at environmental levels, which informs about the origin of the plutonium. In CNA, applying a very simple radiochemistry, detection limits of about $1 \mathrm{fg}\left(10^{-15} \mathrm{~g}\right)$ per sample can be afforded. Since 2007 , a wide variety of studies on ${ }^{239,240} \mathrm{Pu}$ in different environmental compartments (soils, sediments, air-filter samples, sea-water, peat-bogs) have been performed in collaboration with different national and international institutions. On the other hand, in collaboration with the CIEMAT (Spain), a procedure to determine ${ }^{239} \mathrm{Pu}$ in urine samples for internal dosimetry studies had been set up and tested.

Recently, different experiments to set up the ${ }^{236} \mathrm{U}$ measurement technique by AMS have been also performed. Also, radiochemical procedures to extract and purify the uranium fraction have been tested. To date, we have been able to achieve ${ }^{236} \mathrm{U} /{ }^{238} \mathrm{U}$ ratios as low as $10^{-9}$, which compare with the attainable ones on other conventional AMS facilities.

The AMS technique has also proved to be a useful technique to study ${ }^{26} \mathrm{Al}$. The ratio ${ }^{26} \mathrm{Al} /{ }^{10} \mathrm{Be}$ is a very important tool to the study of terrestrial process like erosion in rocks or surface exposure dating by the measurement of exposure. During 2010 the optimization of measurement of ${ }^{26} \mathrm{Al}$ in the $1 \mathrm{MV}$ AMS at CNA facilities has been carried out. In order to obtain the parameters and conditions for making routine measurements of ${ }^{26} \mathrm{Al}$ in this technique, studies of beam transmission, calibration and background values have been carried out in several charge states. The optimization work has led to make recently the first measurements of ${ }^{26} \mathrm{Al}$ in terrestrial samples at CNA, which in this case have been Kaolin ore samples.

While providing an external ${ }^{14} \mathrm{C}$ dating service to users who require it, CNA has conducted also research associated to this nuclide in collaboration with different entities. On this frame, and only as an example, in 2009 it was finished the measurements to obtain a profile of ${ }^{14} \mathrm{C}$ concentration in tree rings from Mexico, which has yielded interesting information about the spatial distribution of ${ }^{14} \mathrm{C}$ 
produced during the nuclear tests of the years 1950-1960. Currently, work is in progress in the determination of ${ }^{14} \mathrm{C}$ in a peat profile from Sweden, an area affected by the so-called Suess effect, consisting of the lower ${ }^{14} \mathrm{C}$ concentration from burning fossil fuels. The magnitude of this Suess effect in the studied area will be assessed.

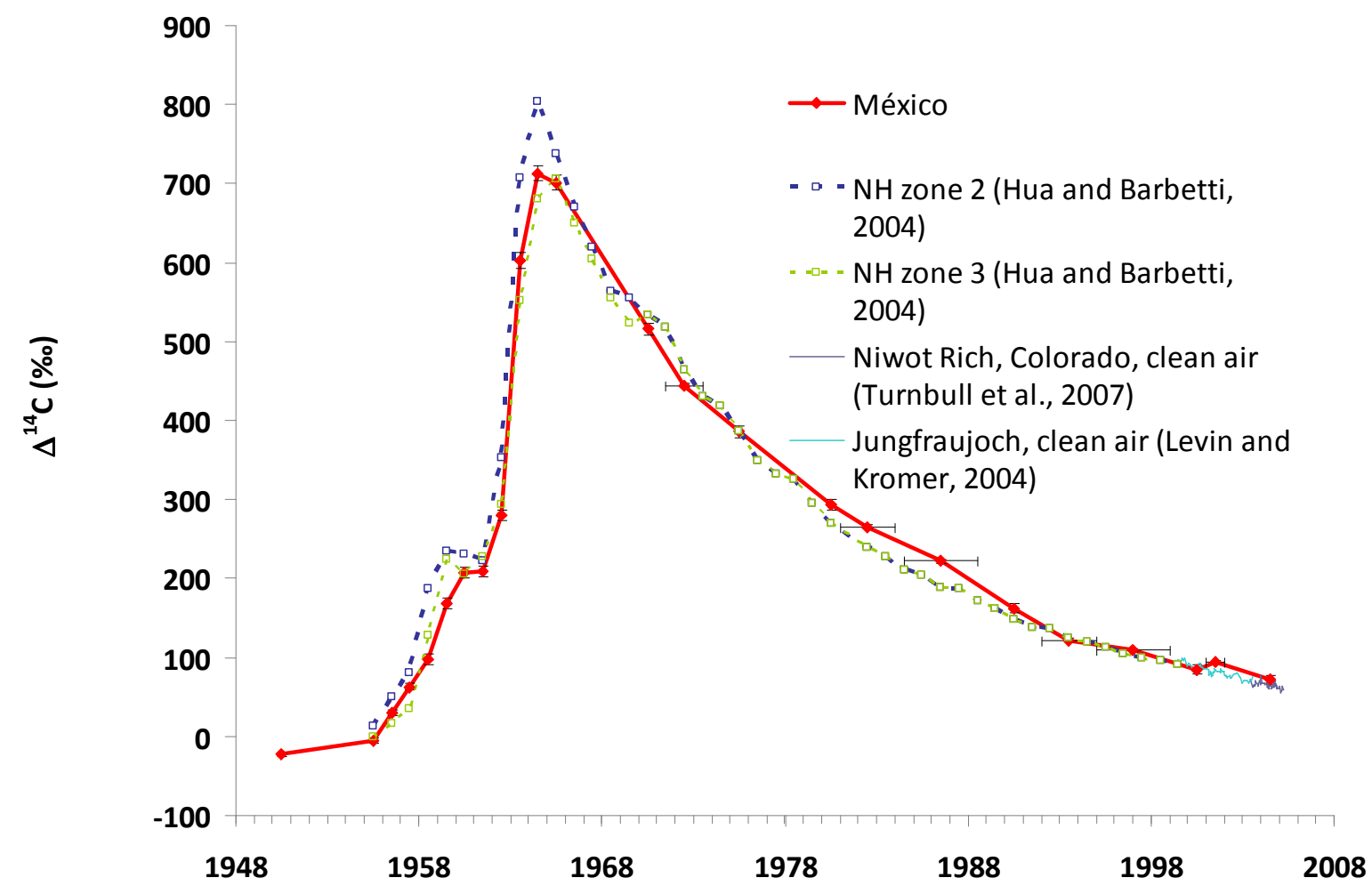

Figure 6: Measurements of $14-\mathrm{C}$ content in tree rings obtained in different geographical zones. The measurements in Mexico tree rings were done at CNA.

Based in the gained experience in AMS, two research projects have been recently developed at CNA funded by ENRESA (Empresa Nacional de Residuos Radiactivos, S.A.) to analyze long-lived radionuclides stored in low and medium-activity nuclear wastes, with special emphasis in ${ }^{129}$ I. The motivation of these studies is the necessity of description for these nuclear wastes to be classified and stored. The first project was based in the development of the technique for the determination of 129

I in these low and medium activity nuclear wastes, such as wet ionic interchange resins and dried muds. Nowadays a new project is being developed looking for the adaptation of the ${ }^{129}$ I methodology set up to more complex matrices.

\section{Biomedical research}

The molecules produced in the CNA radiopharmacy have been used to carry out bio-medical research on animal models. Studies in collaboration with the Medicine Faculty of the University of 
Seville have been carried out to investigate the effect of amitriptilyne, a tricyclic antidepressant, on tumors. This product reduces the antioxidative defenses, leading to death of tumor cells. The evolution of stomach tumors in live mice was followed through the PET images obtained after injected the mice with 18F-FDG, using the micro-PET scanner at CNA. These PET images were combined with CT images, to give a comprehensive picture of the tumor evolution in vivo. These studies were complemented with post-mortem histologic studies.

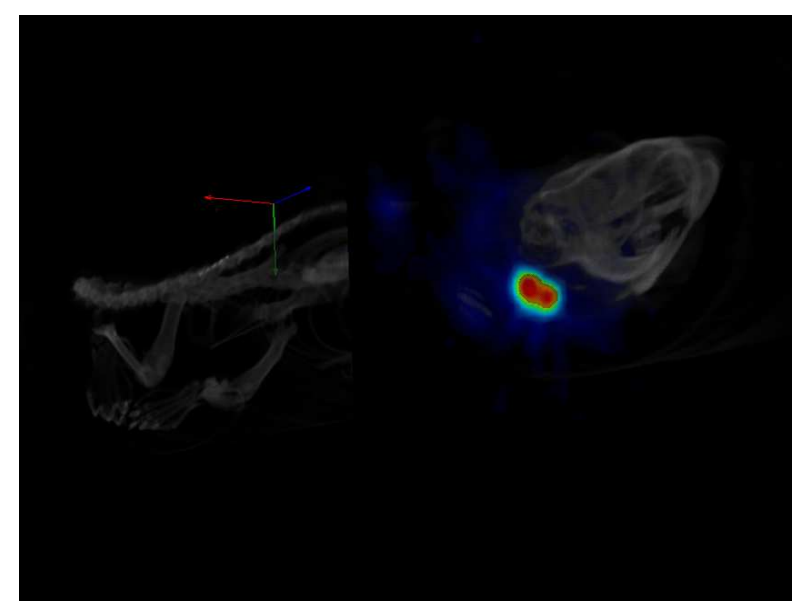

Figure 7: Combined PET-CT image of a rat. The colors correspond to PET image of BF4. The image indicates an abnormal growth of the thyroid gland, due to a tumor.

Brain tumor studies have been also carried out in mice, through multimodal imaging techniques. The PET 18F-FDG image gives an indication of the glucose consumption in the tumor. The PET 18FMISO image indicates whether there is lack of oxygen (hipoxia) in the tumor cells. The CT images obtained allow the precise localization of the tumor, as well as the size determination.

Traumatic damage in the brain has been carried out in rats, in a project in collaboration with the University Hospital "Virgen del Rocío". Intracranial pressure is produced by a Fogarty balloon, which is inflated to a given pressure. PET images are obtained, both with 18F-FDG and 18F-MISO, during the damage, and 24 hours after. The images indicate the evolution of the brain damage and recovery.

The CT equipment at CNA can be used to investigate the preservation of organs for transplants at very low temperature (criopreservation). The challenge is to achieve the vitrification of an organ, by perfusing it with the adequate concentration of Crio-protector agents, such as Dimethilsulfoxide (DMSO). Reliable methods are required to know the DMSO concentration in an organ, prior to freezing. For that purpose, in a collaboration with the Engineering School of Seville University, organs have been simulated using vials containing different concentrations of DMSO, and the CT images have been obtained. It has been found that the contrast in the CT image is proportional to the DMSO concentration. This fact, along with the excellent special resolution $(0.2 \mathrm{~mm})$ make CT imaging a very promising tool for criopreservation studies. 


\section{New facilities at CNA}

We will finish this paper, summarising the new facilities which will be available in the coming months in the centre, opening new fronts in the research to be performed.

CNA has recently acquired a Gammabeam X200 Irradiator, from Bert Theratronics. It has a $12000 \mathrm{Ci}$ source of ${ }^{60} \mathrm{Co}$, fully equipped with TLD dosimetry, a monitor and a probe to measure ambient radiation, and field uniformity sensors. This facility will be installed in a new underground laboratory, allowing irradiation tests required by the European Space Agency, to qualify electronic components for satellite use. Radio-biology studies could also be done, testing the radiation effects in cells, tissues and experimentation animal models. Finally, it will complement the charged particle irradiation studies on materials, solar cells and circuits, already carried out at CNA, with photon irradiation studies.

A new compact accelerator dedicated to ${ }^{14} \mathrm{C}$ AMS measurements will be installed shortly. This system, called MICADAS (Mini Carbon Dating System) has been developed recently at ETH Zurich. It is based on an accelerator of only $200 \mathrm{kV}$, which has proven adequate to perform AMS measurements with light ions. This equipment, along with new grafitization lines that will be installed in the radiocarbon laboratory, will increase the capability to meet the demand of CNA users to perform ${ }^{14} \mathrm{C}$ dating, and also to widen the research of the AMS group in CNA.

A full sized PET-CT scanner, Biograph 64 Slice from Siemens, has recently been acquired. This scanner will allow carrying out clinical trials and human studies at CNA. In this way, the medical community will have the possibility to access to high quality and high resolution images, obtained using the radio-pharmaceuticals produced in the CNA facilities. In particular, it will be possible to carry out studies with ${ }^{11} \mathrm{C}$ based radiopharmaceuticals, such as ${ }^{11} \mathrm{C}$-Choline, ${ }^{11} \mathrm{C}$-Methionine and ${ }^{11} \mathrm{C}$ $\mathrm{PIB}$, that, due to the short lifetime of ${ }^{11} \mathrm{C}$, can only be performed if the Cyclotron and the PET Scanner are in the same place. The PET-CT scanner will open the possibility to carry out studies in oncology, neurology and cardiology.

\section{Reference:}

Memoria de Investigación del CNA, 2009-2010. (CNA Research Report 2009-2010), available at the web-page of the centre: www.cna.us.es

Joaquín Gómez Camacho, Director of CNA, gomez@us.es

Rafael García Tenorio, ViceDirector of CNA, gtenorio@us.es 